\title{
Vacinação contra o rotavírus nos cuidados de saúde primários
}

Carla Laranjeira,* Rita Eiriz,* Paulo Santos*

\section{RESUMO}

Objetivos: Determinar a cobertura da vacina anti-rotavírus na Unidade de Saúde Familiar S. João do Porto e verificar se a vacinação está associada a resultados clínicos (número de episódios, número de dias de doença e número de dias de absentismo laboral dos cuidadores). Tipo de Estudo: Realizou-se um estudo analítico transversal.

Local: Unidade de Saúde Familiar de S. João do Porto.

População: Crianças com idades compreendidas entre os 6 meses e os 5 anos.

Métodos: Aplicou-se um questionário por via telefónica aos pais de 500 crianças, selecionadas por técnica de amostragam aleatória nos inscritos nas listas da Unidade de Saúde. Para a análise univariada usaram-se os testes do qui-quadrado, Mann-Whitney U e Mantel Cox. Um modelo de regressão logística foi usado para análise multivariada. Aceitou-se um erro alfa de 0,05.

Resultados: A proporção de cobertura vacinal foi de 55,1 \% (IC95\%: 48,8-61,4\%). A frequência do infantário antes dos 6 meses associou-se a uma maior cobertura vacinal $(p=0,015)$, com uma mediana de idade de entrada no infantário de 11 meses no grupo vacinado versus 24 meses no não vacinado $(p=0,007)$. Após ajustamento para as principais variáveis, a variação do número de episódios de diarreia entre os grupos vacinado e não vacinado apresentou um odds ratio de 0,933 (IC95\%: 0,539-1,613).

Conclusões: Pouco mais de metade da população aderiu à vacinação anti-rotavírus. Neste estudo não se demonstra benefício clinicamente evidente na diminuição do número de episódios de diarreia, na duração dos episódios e no absentismo laboral dos cuidadores em relação com a vacinação.

Palavras-chave: Vacina Rotavirus; Eficácia; Saúde Infantil.

\section{INTRODUÇÃO}

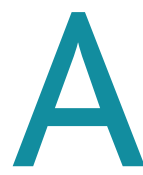
gastroenterite aguda (GEA) é uma patologia comum nos primeiros anos de vida em todo o mundo. Na Europa estima-se que cada criança com menos de 3 anos de idade tenha, por ano, cerca de 0,9-1,9 episódios de GEA. ${ }^{1-3}$

Os vírus são os agentes etiológicos mais comuns. Predominam os rotavírus e calcivírus, e com menor frequência, o adenovírus e $\mathrm{o}$ astrovírus.

Há poucos estudos na Europa que tenham avaliado a incidência da gastroenterite e os agentes causais. Da evidência disponível, sabe-se que o rotavírus é o agente causal mais frequente, podendo ser o responsável até cerca de $35 \%$ dos casos. A infeção por rotavírus é frequente nas crianças com menos de cinco anos de idade, sendo que as crianças com menos de dois anos de idade têm pelo menos um episódio durante este período.

Nos países desenvolvidos a maioria das crianças não necessita de hospitalização e a mortalidade é rara, mas o im-

*Médica interna de Medicina Geral e Familiar.

* Médica interna de Medicina Geral e Familiar.

* Médico assistente de Medicina Geral e Familiar.

USF S. João do Porto - ACeS Porto Ocidental pacto da doença é significativo no bem estar da criança e no absentismo laboral dos pais. ${ }^{4}$

Neste contexto foram introduzidas duas vacinas anti-rotavírus em Portugal em 2006, com o objetivo de reduzir a morbilidade por GEA, sobretudo até aos dois anos de idade, altura em que ocorrem mais episódios por gastroenterite e que podem ser potencialmente mais graves.

Têm sido publicados vários estudos que demonstram a eficácia da vacina na redução da mortalidade (em países de África e América do Sul) e na diminuição da incidência de casos graves (Europa e Estados Unidos da América). ${ }^{4-27}$

Em relação à Europa, foram desenvolvidos vários estudos de custo eficácia para decidir sobre a introdução da vacina no esquema universal de cada país. Os parâmetros considerados variaram de país para país, daí que em alguns casos, como a Holanda, ${ }^{28}$ a Bélgica ${ }^{29} \mathrm{e}$ a Itália, ${ }^{30}$ a vacina mostrou-se custo-eficaz. Por outro lado, no Reino Unido, ${ }^{31}$ Irlanda, ${ }^{32}$ França $^{33} \mathrm{e}$ Espanha, ${ }^{34}$ a vacina não se mostrou custo-eficaz. As conclusões destes estudos podem variar conforme se considere o bem-estar da criança e o absentismo dos cuidadores e não só os custos diretos em recursos de saúde e mortes para os respetivos Estados.

Em relação aos cuidados de saúde primários dispõe-se de 
menor conhecimento sobre a eficácia da vacina, nomeadamente nos casos mais ligeiros de gastroenterite e do impacto no absentismo laboral dos pais.

Em Portugal não existem estudos publicados sobre a eficácia da vacina, há apenas dados regionais sobre os serótipos predominantes em Braga e Coimbra em 2007, em crianças que recorreram ao Serviço de Urgência do Hospital de Braga e Pediátrico de Coimbra. Há ainda um estudo de base hospitalar (vários hospitais do continente e ilhas incluídos) de 2008 que mostrou que a GEA foi motivada por rotavírus em $27,8 \%$ das crianças com menos de cinco anos de idade observadas no Serviço de urgência. ${ }^{35-37}$

O objetivo deste trabalho foi determinar a proporção de crianças vacinadas contra o rotavírus na USF S. João do Porto. Secundariamente procurou-se verificar se a vacinação estava associada a resultados clínicos traduzidos pelo número de episódios ou dias de diarreia e pelo absentismo dos cuidadores.

\section{MÉTODOS}

Realizou-se um estudo observacional analítico transversal numa amostra de crianças com idades compreendidas entre os 6 meses e os 5 anos da USF S. João do Porto.

Sendo desconhecida a taxa de cobertura vacinal da população em estudo, para o cálculo da dimensão amostral assumiu-se uma proporção provável de 50\%. Considerando uma população de 2000 utentes nesta faixa etária e aceitando uma margem de erro de $5 \%$ para um intervalo de confiança de 95\%, o cálculo do tamanho amostral resultou num mínimo de 323 crianças. Prevendo uma taxa de respondedores de cerca de dois terços, assumiu-se inclusão de 500 crianças, selecionadas por aleatorização simples a partir da da base de dados da unidade de saúde, utilizando a aplicação informática Research randomizer. ${ }^{31}$

Os dados foram colhidos por questionário aplicado através de entrevista telefónica, tendo sido contactados os pais ou tutores das crianças até um total de 5 tentativas em vários horários, incluindo o fim de semana. O questionário foi desenvolvido e aplicado pelos investigadores. Foi pedido o consentimento oral aos pais, garantindo-se a confidencialidade e o rigor ético no tratamento dos dados. O estudo teve a aprovação prévia da Comissão de Ética para a Saúde da ARS Norte.

As variáveis estudadas incluíram a vacinação anti-rotavírus, o tipo de vacina e o número de doses, o género, a idade, o tempo de gestação (semanas), o peso ao nascimento, a presença de imunodeficiência, a presença de comorbilidades, o tempo de amamentação materna (em meses), o início e permanência em infantário, o número de episódios de
GEA e a sua duração, e o absentismo laboral dos cuidadores em dias. Definiu-se como episódio de GEA a presença de mais de três dejeções de consistência mole ou mais abundante que o normal, associadas ou não a vómitos ou febre. Foi considerada vacinada a criança cujos tutores responderam ter completado o esquema vacinal prescrito pelo profissional de saúde. Para eliminar um possível viés de memória, foi pedido aos pais durante o contato telefónico para consultar o boletim de vacinação e foram dadas instruções para a pesquisa das vacinas em questão.

Os dados recolhidos foram anonimizados e registados numa base de dados informática Microsoft Office Excel $2007 \AA$ e tratados estatisticamente no SPSS® versão 20.0.

$\mathrm{Na}$ análise estatística foram usadas medidas de frequência, tendência central e dispersão. Os intervalos de confiança foram calculados pelo método de Wald. Para testar a associação entre variáveis categóricas foi utilizado o teste de qui-quadrado, nas variáveis contínuas foi utilizado o teste de Mann-Whitney U e nas variáveis temporais utilizou-se o teste de Log Rank (Mantel Cox). A análise multivariada foi realizada recorrendo a um modelo de regressão logística. Aceitou-se um erro alfa de 0,05 .

\section{RESULTADOS}

Obteve-se resposta a 243 questionários, relativos ao mesmo número crianças (53,5\% do sexo feminino), correspondendo a uma taxa de resposta de 48,6\%. A mediana da idade situou-se nos 40 meses (distância interquartil=27 meses), variando entre os 8 e os 64 meses à data da colheita dos dados.

A cobertura vacinal nesta população foi de 55,1\% (IC95\%: 48,8-61,4\%). Considerou-se que o esquema de vacinação estava completo se as crianças tinham efetuado três doses de vacina pentavalente e duas da monovalente. Foram consideradas vacinadas as crianças que cumpriram o esquema anterior. Nas crianças que completaram o esquema vacinal, $69,2 \%$ fizeram-no com a vacina pentavalente. Três casos não completaram as três doses da vacinação pentavalente e um caso iniciou a vacina pentavalente e fez uma segunda dose da monovalente. No quadro 1 descrevem-se as variáveis intrínsecas à criança que poderiam estar relacionadas com a decisão de vacinar. Apenas a decisão de integrar os filhos antes dos seis meses de idade no infantário se associava significativamente a uma maior proporção de vacinação $(25,4 \%$ para $12,8 \%, p=0,015)$, sendo evidente que o grupo dos vacinados entrava no infantário significativamente mais precocemente, com uma mediana de idade de 11 meses versus 24 meses no grupo dos não vacinados ( $\mathrm{p}=0,007)$.

No grupo das crianças vacinadas, 61,9\% (IC95\%: 53,2- 


\begin{tabular}{|c|c|c|c|c|}
\hline \multicolumn{2}{|l|}{$\mathrm{N}=\mathbf{2 4 3}$} & \multirow{2}{*}{$\begin{array}{l}\text { Vacinados } \\
15(50,0 \%)\end{array}$} & \multirow{2}{*}{$\begin{array}{r}\text { Não vacinados } \\
15(50,0 \%)\end{array}$} & \multirow{3}{*}{\begin{tabular}{|c|}
$\mathbf{P}$ \\
$0,545^{*}$
\end{tabular}} \\
\hline Prematuridade & Sim & & & \\
\hline & Não & $119(55,9 \%)$ & $94(44,1 \%)$ & \\
\hline \multirow[t]{2}{*}{ Baixo peso ao nascimento } & Sim & $17(58,6 \%)$ & $12(41,4 \%)$ & \multirow[t]{2}{*}{$0,688^{*}$} \\
\hline & Não & $117(54,7 \%)$ & $97(45,3 \%)$ & \\
\hline \multirow{2}{*}{$\begin{array}{l}\text { Presença de comorbilidade nas } \\
\text { crianças }\end{array}$} & Sim & $9(52,9 \%)$ & $8(47,1 \%)$ & \multirow[t]{2}{*}{$0,850^{*}$} \\
\hline & Não & $125(55,3 \%)$ & $101(44,7 \%)$ & \\
\hline \multirow[t]{2}{*}{ Amamentação (à saída do hospital) } & Sim & $126(55,5 \%)$ & $101(44,5 \%)$ & \multirow[t]{2}{*}{$0,669 *$} \\
\hline & Não & $8(50,0 \%)$ & $8(50,0 \%)$ & \\
\hline \multirow[t]{2}{*}{ Amamentação (>3 meses) } & Sim & $101(54,3 \%)$ & $85(45,7 \%)$ & \multirow[t]{2}{*}{$0,633^{*}$} \\
\hline & Não & $33(57,9 \%)$ & $24(42,1 \%)$ & \\
\hline \multicolumn{2}{|l|}{$\begin{array}{l}\text { Tempo de amamentação (mediana } \\
\text { em meses; DP) }\end{array}$} & $6,00( \pm 0,72)$ & $5,00( \pm 0,45)$ & $0,896 * *$ \\
\hline Frequência de infantário antes dos & Sim & $34(70,8 \%)$ & $14(29,1 \%)$ & $0,015^{*}$ \\
\hline 6 meses & Não & $100(51,3 \%)$ & $95(48,7 \%)$ & \\
\hline \multicolumn{2}{|l|}{$\begin{array}{l}\text { Idade de entrada para o infantário } \\
\text { (mediana em meses; DP) }\end{array}$} & $11,00( \pm 2,67)$ & $24,00( \pm 3,17)$ & $0,007 * *$ \\
\hline
\end{tabular}

*teste do qui-quadrado; **teste de Log Rank (Mantel Cox)

$70,2 \%)$ apresentaram pelo menos um episódio de GEA versus $62,4 \%$ (IC95\%: 52,6-71,5\%) no grupo não vacinado, representando um odds ratio de 0,981 (IC95\%: 0,583-1,653; $\mathrm{p}=0,943)$.

A média de episódios foi de 2,8 (IC95\%: 2,2-3,3) nas não vacinadas e 2,7 (IC95\%: 2,0-3,3) nas vacinadas ( $\mathrm{p}=0,408)$. O absentismo dos cuidadores foi de 1,6 dias (IC95\%: 0,9-2,3) nas não vacinadas e 1,1 dias (IC95\%: 0,7-1,5) nas vacinadas $(\mathrm{p}=0,520)$.

Ajustando a análise para género e idade da criança, baixo peso ao nascimento, prematuridade, presença de comorbilidade infantil, amamentação por mais de três meses e entrada no infantário antes dos seis meses, não se verifica uma diminuição da GEA em relação com a vacinação (odds ratio = 0,933; IC95\%: 0,539-1,613).

\section{DISCUSSÃO}

A cobertura vacinal obtida na USF S. João do Porto foi de 55 \%, não sendo conhecidos outros valores em Portugal. O relatório da UNICEF de 2011 relata uma taxa de cobertura a nível mundial de $9 \%$, variando entre os $2 \%$ da região africana e os $66 \%$ nas Américas. ${ }^{38}$

Uma vacina é um produto que, administrado no indivíduo, vai modular o seu sistema imunológico de forma a prevenir o aparecimento da doença, reduzindo a sua incidência na população. A avaliação desta tecnologia de saúde passa pela apreciação cientificamente validada da sua eficácia, da sua segurança, da relação de custo-benefício e dos aspetos éticos, sociais e psicológicos envolvidos. Sob este ponto de vista, interessa saber a evolução após a introdução do procedimento no sentido de se proceder a uma avaliação retrospetiva da atuação médica.

Neste sentido, avaliou-se comparativamente os grupos de crianças vacinadas e não vacinadas, não se observando diferenças estatisticamente significativas quer no número de episódios quer na duração de GEA.

Mesmo na análise dos dados até ao segundo ano de vida, altura em que os episódios são potencialmente mais frequentes e graves, não se verificaram diferenças significativas. De igual modo, também não se registou uma diminuição no absentismo laboral dos cuidadores.

Da revisão da literatura atual, destaca-se um estudo efetuado nos Estados Unidos da América no âmbito dos cuidados de saúde primários, que comparou o número de episódios de GEA nos anos de 2007 e 2008. Neste estudo observou-se uma redução de $56 \%$ para $46 \%$ no número de episódios no ano de 2007, mas no ano seguinte não foi observada diferença significativa na incidência da GEA, ${ }^{39}$ colocando-se a hipótese de um possível efeito de imunidade de grupo, de variação na infecciosidade do agente ou de um desvio para outros patogénios não presentes na vacina. No estudo, a hipótese da imunidade de grupo não parece plausível pela baixa taxa de adesão à vacina. A questão das variações cíclicas na incidência e patogenicidade do rotavírus foi contornada ao serem incluídos vários anos de nascimento. O desvio epidemiológico para outros agentes é possível mas não foi objetivo neste estudo.

Este estudo foi inovador na forma de determinação da taxa de vacinação anti-rotavírus em Portugal. A vacina não está incluída no Programa Nacional de Vacinação e os regis- 
tos vacinais nos cuidados de saúde primários podem não refletir com rigor a proporção de crianças vacinadas por um défice nos registos no programa informático. Por outro lado, a acessibilidade à vacina depende de questões financeiras não desprezíveis, na medida em que comporta um custo de aquisição que não é diluído através da comparticipação pelo Serviço Nacional de Saúde. A amostragem com base nos inscritos na USF permitiu ultrapassar este possível viés.

A proporção de respondedores foi baixa e pode condicionar a extrapolação para a população inscrita na USF, embora tenha sido feito um esforço para concretizar os contactos. A principal dificuldade encontrada foi a dos contactos telefónicos estarem desatualizados na base de dados da USF.

Para verificar a existência de um viés de memória dos pais, efetuou-se a análise por categorias de idade que não mostrou diferenças entre os grupos, o que parece não interferir nas conclusões. Por outro lado, foi explicado a cada cuidador, de forma rigorosa e objetiva, a definição de gastroenterite aguda, para uma melhor operacionalização dos dados.

Seria interessante alargar este estudo para responder a um objetivo principal de efetividade na prática clínica, com uma metodologia prospetiva que permitisse ajustar os viéses identificados.

A vacinação é um tema central na Medicina Geral e Familiar. Os médicos de família são um pilar fundamental na informação e adesão à vacinação. Neste sentido, o aumento de conhecimento nesta área permite uma melhor prestação de cuidados e recursos.

\section{REFERÊNCIAS BIBLIOGRÁFICAS}

1. Secção de gastroenterologia e nutrição pediátrica. Vacina contra o rotavírus: recomendações - 2009. Lisboa: Sociedade de Infecciologia Pediátrica; 2009.

2. Guarino A, Albano F, Ashkenazi S, Gendrel D, Hoekstra JH, Shamir R, et al. Evidence-based guidelines for the management of acute gastroenteritis in children in Europe. European Society for Paediatric Gastroenterology, Hepatology, and Nutrition/European Society for Paediatric Infectious Diseases.J Pediatr Gastroenterol Nutr. 2008;46Suppl2:S81-4.

3. Matson DO. Rotavirus vaccines. In UpToDate [database]; 2010 [cited 2010 Oct 11]. Available from: http://www.uptodate.com

4. Soares-Weiser K, Maclehose H, Bergman H, Ben-Aharon I, Nagpal S, Goldberg $E$, et al. Vaccines for preventing rotavirus diarrhoea: vaccines in use. Cochrane Database Syst Rev. 2012;11:CD008521.

5. Shatsky M. Rotavirus vaccine, live, oral, pentavalent (RotaTeq) for prevention of rotavirus gastroenteritis. Am Fam Physician. 2006;74 (6):1014-5.

6. Ruiz-Palacios GM, Pérez-Schael I, Velázquez FR, Abate H, Breuer T, Clemens SC, et al. Safety and efficacy of an attenuated vaccine against severe rotavirus gastroenteritis. N Engl J Med. 2006;354(1):11-22.

7. Vesikari T, Karvonen AV, Majuri J, Zeng SQ, Pang XL, Kohberger R, et al. Safety, efficacy, and immunogenicity of 2 doses of bovine-human (UK) and rhesusrhesus-human rotavirus reassortant tetravalent vaccines in Finnish children. J Infect Dis. 2006;194(3):370-6.

8. Vesikari T, Karvonen A, Prymula R, Schuster V, Tejedor JC, Cohen R, et al. Effica- cy of human rotavirus vaccine against rotavirus gastroenteritis during the first 2 years of life in European infants: randomised, double-blind controlled study. Lancet. 2007;370(9601):1757-63.

9. Linhares AC, Velázquez FR, Pérez-Schael I, Sáez-Llorens X, Abate H, Espinoza F, et al. Efficacy and safety of an oral live attenuated human rotavirus vaccine against rotavirus gastroenteritis during the first 2 years of life in Latin American infants: a randomised, double-blind, placebo-controlled phase III study. Lancet. 2008;371 (9619):1181-9.

10. Tate JE, Curns AT, Cortese M,Weintraub ES, Hambidge S, Zangwill KM, et al. Burden of acute gastroenteritis hospitalizations and emergency department visits in US children that is potentially preventable by rotavirus vaccination: a probe study using the now-withdrawn rotashield vaccine. Pediatrics. 2009; 123(3):744-9.

11. Committee on Infectious Diseases, American Academy of Pediatrics. Prevention of rotavirus disease: updated guidelines for use of rotavirus vaccine. Pediatrics. 2009;123(5):1412-20.

12. De Vos B, Han HH, Bouckenoghe A, Debrus S, Gillard P, Ward R, et al. Live attenuated human rotavirus vaccine, RIX4414, provides clinical protection in infants against rotavirus strains with and without shared $G$ and $P$ genotypes: integrated analysis of randomized controlled trials. Pediatr Infect Dis J. 2009; 28(4):261-6.

13. Lambert SB, Faux CE, Hall L, Birrell FA, Peterson KV, Selvey CE, et al. Early evidence for direct and indirect effects of the infant rotavirus vaccine program in Queensland. Med J Aust. 200; 191(3): 157-60.

14. Armah GE, Sow SO, Breiman RF, Dallas MJ, Tapia MD, Feikin DR, et al. Efficacy of pentavalent rotavirus vaccine against severe rotavirus gastroenteritis in infants in developing countries in sub-Saharan Africa: a randomised, double-blind, placebo-controlled trial. Lancet. 2010;376(9741):606-14.

15. RicharsonV,Hernández-Pichardo J, Quintalar-Solares M, Esparza-Aguilar M, Johnson B, Gómez-Altamirano, et al. Effect of rotavirus vaccination on death from childhood diarrhea in Mexico. N Engl J Med. 2010;362(4):299-305.

16. Zaman K, Dang DA, Victor JC, Shin S, Yunus M, Dallas MJ, et al. Efficacy of pentavalent rotavirus vaccine against severe rotavirus gastroenteritis in infants in developing countries in Asia: a randomised, double-blind, placebo-controlled trial. Lancet. 2010;376(9741):615-23.

17. Madhi SA, Cunliffe NA, Steele D, Witte D, Kirsten M, Louw C, et al. Effect of human rotavirus vaccine on severe diarrhea in African infants. N Engl J Med. 2010;362(4):289-98.

18. Palma O, Cruz L, Ramos H, de Baires A, Villatoro N, Pastor D, et al. Effectiveness of rotavirus vaccination against childhood diarrhoea in El Salvador: case-control study. BMJ. 2010;340:c2825.

19. Clark HF, Lawley D, Matthijnssens J, DiNubile MJ, Hodinka RL. Sustained decline in cases of rotavirus gastroenteritis presenting to the Children's Hospital of Philadelphia in the new rotavirus vaccine era. Pediatr Infect Dis J. 2010;29 (8):699-702.

20. Vesikari T, Karvonen A, Ferrante SA, Kuter BJ, Ciarlet M. Sustained efficacy of the pentavalent rotavirus vaccine, RV5, up to 3.1 years following the last dose of vaccine. Pediatr Infect Dis J. 2010;29 (10): 957-63.

21. Field EJ, Vally H, Grimwood K, Lambert SB. Pentavalent rotavirus vaccine and prevention of gastroenteritis hospitalizations in Australia. ediatrics. 2010;126 (3): e506-12

22. Bégué RE, Perrin $K$. Reduction in gastroenteritis with the use of pentavalent rotavirus vaccine in a primary practice. Pediatrics. 2010;126(1):e40-5.

23. Curns AT, Steiner CA, Barrett M, Hunter K, Wilson E, Parashar UD. Reduction in acute gastroenteritis hospitalizations among US children after introduction of rotavirus vaccine: analysis of hospital discharge data from 18 US states. J Infect Dis. 2010;201(11):1617-24.

24. Cortese MM, Tate JE, Simonsen L, Edelman L, Parashar UD. Reduction in gastroenteritis in United States children and correlation with early rotavirus vaccine uptake from national medical claims databases. Pediatr Infect Dis J. 
2010;29(6):489-94.

25. Wang FT, Mast TC, Glass RJ, Loughlin J, Seeger JD. Effectiveness of the pentavalent rotavirus vaccine in preventing gastroenteritis in the United States. Pediatrics. 2010;125(2):e208-13.

26. Boom JA, Tate JE, Sahni LC, Rench MA, Hull JJ, Gentsch JR, et al. Effectiveness of pentavalent rotavirus vaccine in a large urban population in the United States. Pediatrics. 2010;125(2):e199-207.

27. Paulke-Korinek M, Rendi-Wagner P, Kundi M, Kronik R, Kolaritsch H. Universal mass vaccination against rotavirus gastroenteritis: impact on hospitalization rates in austrian children. Pediatr Infect Dis J. 2010;29(4):319-23.

28. Bilcke J, Van Damme P, Beutels P. Cost-effectiveness of rotavirus vaccination: exploring caregiver(s) and "no medical care" disease impact in Belgium. Med Decis Making. 2009;29(1):33-50.

29. Giammanco MD, Coniglio MA, Pignato S, Giammanco G. An economic analysis of rotavirus vaccination in Italy. Vaccine. 2009;27(29):3904-11.

30. Jit $M, E d m u n d s W J$. Evaluating rotavirus vaccination in England andWales. Part II:The potential cost-effectiveness of vaccination. Vacine. 2007;25(20):3971-9.

31. Tilson L, Jit M, Schmitz S, Walsh C, Garvey P, McKeown P, et al. Cost-effectiveness of universal rotavirus vaccination in reducing rotavirus gastroenteritis in Ireland. Vacine. 2011;29(43):7463-73.

32. Huet F, Largeron N, Trichard M, Miadi-Fargier H, Jasso-Mosqueda G. Burden of paediatric rotavirus gastroenteritis and potential benefits of a universal rotavirus vaccination programme with RotaTeq in France.Vaccine. 2007;25(34):6348-58 .

33. Imaz I, Rubio B, Cornejo AM, González-Enríquez J. Cost-utility analysis of the introduction of universal infant rotavirus vaccination in Spain. Prev Med. 2014; 61: 116-21.

34. Rodrigues F, Iturriza M, Gray J, Januário L, Lemos L. Epidemiology of rotavirus in Portugal: G9 as a major cause of diarrhoea in non-hospitalised children. J Clin Virol. 2007;40 (3): 214-7.

35. Antunes H, Afonso A, Iturriza M, Martinho I, Ribeiro C, Rocha S, et al. G2P[4] the most prevalent rotavirus genotype in 2007 winter season in an European non- vaccinated population. J Clin Virol. 2009;45 (1):76-8.

36. Rodrigues F, Iturriza-Gómara M, Marlow R, Gray J, Nawaz S, Januário L, et al. The evolving epidemiology of rotavirus gastroenteritis in central Portugal with modest vaccine coverage. J Clin Virol. 2013;56(2):129-34.

37. Urbaniak $G$, Plous $C$. Research randomizer (version 2.0) [computer software]. Randomizer; 2007 [cited 2011 Mar 1]. Available from: http://www.randomizer.org .

38. World Health Organization, United Nations Children's Fund. WHO/UNICEF coverage estimates. Geneva: WHO; 2013 [cited 2013 Jun 27]. Available from: http://www.who.int/entity/immunization_monitoring/data/coverage_estimates_series.xls

39. Nolan SM, Prasad P, Fiks AG, Zaoutis T, TenHave TR, Coffin SE. Effect of rotavirus vaccine on reducing acute gastroenteritis in a large outpatient pediatric network. Arch Pediatr Adolesc Med. 2012;166(3):232-9.

\section{CONFLITO DE INTERESSES}

Os autores declaram não haver conflito de interesses no presente estudo. A instituição empregadora não tem qualquer interesse ou conflito financeiro relacionado com os resultados ou conclusões do manuscrito.

\section{FINANCIAMENTO DO ESTUDO}

O financiamento do estudo ficou a cargo dos autores. Os inquéritos telefónicos foram efetuados com recurso a meios de comunicação da responsabilidade dos autores.

\section{ENDEREÇO PARA CORRESPONDÊNCIA}

Carla Laranjeira

USF S. João do Porto, Rua Miguel Bombarda, 234, 4050-377 Porto

E-mail: cristinalaranjeira@sapo.pt

Recebido em 11-07-2013

Aceite para publicação em 21-01-2014

Artigo escrito ao abrigo do novo acordo ortográfico.

\section{ABSTRACT}

\section{EFFICACY OF ROTAVIRUS VACCINE IN PRIMARY CARE}

Aim: To determine the coverage of anti-rotavirus immunization in the Family Health Unit of S. João do Porto and to test the association of immunization with clinical outcomes (number of episodes of acute gastroenteritis, their duration, and days of work lost by the parents). Type of study: Cross-sectional

Setting: Family Health Unit S. João do Porto

Population: Children aged between 6 months and 5 years old

Methods: Parents of 500 children, randomly selected from the list of patients of the Health Unit, were contacted by telephone for this survey. The chi-square test, Mann-Whitney $\mathrm{U}$ test, and Mantel-Cox test were used for univariate analysis of the association between the predictor and outcome variables. Multivariate analysis was performed using a logistic regression model. Significance was set at the of 0.05 level. Results: Immunization coverage by rotavirus vaccine in this population was $55.1 \%$ (95\% $\mathrm{Cl}: 48.8-61.4 \%$ ). Entry into kindergarten before 6 months of age was associated with higher vaccination coverage $(p=0.015)$. The median age for entry to kindergarten was lower in the vaccinated group ( 11 vs 24 months, $\mathrm{p}=0.007$ ). After adjusting for gender, age, low birth weight, prematurity, comorbidity, breastfeeding for over 3 months, and attending kindergarten before 6 months of age, the odds ratio for the efficacy of the vaccine was estimated to be 0.933 (95\%Cl: 0.539-1.613).

Conclusions: Over half of this population received rotavirus immunization. This study did not find evidence of clinical efficacy of the vaccine, neither in terms of number of episodes of diarrhea, their duration, nor in work days lost by caregivers. These findings raise questions about the value of rotavirus vaccine. 